\title{
Revisit Phase Separation of the Two-Dimensional $t-J$ model by the Power-Lanczos Method
}

\author{
C.T. Shih ${ }^{1}$, Y.C. Chen ${ }^{2}$, and T.K. Lee ${ }^{3,4}$ \\ ${ }^{1}$ Division of Science Application, National Center for High-Performance Computing, Hsinchu, Taiwan \\ ${ }^{2}$ Dept. of Physics, Tunghai Univ., Taichung, Taiwan \\ 3 Inst. of Physics, Academia Sinica, Nankang, Taipei, Taiwan \\ ${ }^{4}$ National Center for Theoretical Sciences, Hsinchu, Taiwan
}

(November 3, 2018)

\begin{abstract}
The power-Lanczos (PL) method is one kind of Green's function Monte-Carlo simulation, which is improved by Lanczos iterations. The ground state energies of strongly-correlated models can be evaluated by this method quite accurately. In this report, the boundary of phase separation (PS) of the two-dimensional $t-J$ model is investigated by the power-Lanczos method and Maxwell construction. The energies are compared with the results evaluated by other methods. Our conclusion is that there is no phase separation for $J / t \leq 0.4$.
\end{abstract}

PACS numbers: 74.20.-z, 71.27.+a, 74.25.Dw

\section{INTRODUCTION}

It is believed that several key features of the hightemperature superconductors (HTSC) can be described by the two-dimensional (2D) $t-J$ model on square lattices. The Hamiltonian is:

$$
H=-t \sum_{<i, j>\sigma}\left(\tilde{c}_{i \sigma}^{+} \tilde{c}_{j \sigma}+h . c .\right)+J \sum_{<i, j>}\left(\mathbf{S}_{i} \cdot \mathbf{S}_{j}-\frac{1}{4} n_{i} n_{j}\right)
$$

where $\langle i, j\rangle$ is the nearest-neighbor pairs and

$$
\tilde{c}_{i \sigma}=c_{i \sigma}\left(1-n_{i,-\sigma}\right)
$$

One of the important questions on this model is whether the density distribution of charge carriers in the $\mathrm{CuO}_{2}$ planes is uniform or not. There are several types of non-uniform phase such as macroscopic phase separation which is separated into a hole-free antiferromagnetif $(\mathrm{AF})$ region and a hole-rich regiont, and the stripel phase which holes form domain walls separating AF regions. The ground state is determined by the competition of the kinetic $(t)$ and exchange $(J)$ terms. The $t$ term favors the uniform phase to minimize the kinetic energy, while $J$ term tend to attract the electrons together to lower the magnetic energy.

PS state and superconductivity are closely related. Some groups argued that the driving mechanism of st perconductivity is the same as that of phase separation 6 or superconductivity comes from the frustrated phase separationt. On the other hand, if PS occurs in the physical regime, the system will become insulating, and of course, no superconductivity. Hence it is extremely important to determine the phase separation boundary of the 2D $t-J$ model to resolve these issues.

Experimentally, phase separation of the superconducting $\mathrm{La}_{2} \mathrm{CuO}_{4+\delta}$ compound are pserved by neutron powder diffraction $8,{ }^{139} \mathrm{La}$ NMR 10 and magnetic susceptibility $11 . \mathrm{La}_{2} \mathrm{CuO}_{4+\delta}$ phase separates for $0.01 \leq$ $\delta \leq 0.06$ below $\mathrm{T}_{p s} \approx 300 K$ into the nearly stoichiometric antiferromagnetic $\mathrm{La}_{2} \mathrm{CuO}_{4+\delta_{1}}$ with $\delta_{1}$ less than 0.02 and Néel temperature $T_{N} \approx 250 K$, and a metallic superconducting oxygen-rich phase $\mathrm{La}_{2} \mathrm{CuO}_{4+\delta_{2}}$ with $\delta_{2} \approx$ 0.06 and superconducting transition temperature $T_{c} \approx$ $34 K$. In a recent transmission electron microscopy experiment, an incommensurate modulation was observed directly in a phase separated $\mathrm{Cu}$-rich $\mathrm{La}_{2} \mathrm{CuO}_{4.003}$ 12. J. $\mathrm{H}$. Cho et al. 13 measured the magnetic susceptibility of the $\mathrm{Sr}$ doped compound $\mathrm{La}_{2-x} \mathrm{Sr}_{x} \mathrm{CuO}_{4+\delta}$. For $x \leq 0.03$, they also found macroscopic phase separation into superconducting $\mathrm{La}_{2-x} \mathrm{Sr}_{x} \mathrm{CuO}_{4+\delta^{\prime}}\left(\delta^{\prime} \approx 0.08\right)$ and nonsuperconducting $\mathrm{La}_{2-x} \mathrm{Sr}_{x} \mathrm{CuO}_{4+\delta^{\prime \prime}}\left(\delta^{\prime \prime} \approx 0.00\right)$ phases. For $x \leq 0.02$, the later phase is antiferromagnetically ordered. The long range order disappears and the phase becomes spin-glass-like for $0.02 \leq x \leq 0.028$. The doped holes in the AF (or spin-glass) phase $\mathrm{La}_{2-x} \mathrm{Sr}_{x} \mathrm{CuO}_{4+\delta^{\prime \prime}}$ $\left(0 \leq x \leq 0.03, \delta^{\prime \prime} \approx 0.00\right)$ condense into walls separating microscopic undoped domains. The phase becomes inhomogeneous electronically and magnetically.

On the other hand, the well known $\mathrm{La}_{2-x} \mathrm{Sr}_{x} \mathrm{CuO}_{4}$ superconductor does not phase separate macroscopically because the $\mathrm{Sr}^{+2}$ ions are immobile and can not phase separate along with the doped holes. In 1989, A. Weidinger et al. 4 observed coexistence of magnetic ordering and superconductivity by muon spin rotation $\left(\mu^{+} \mathrm{SR}\right)$ experiments performed on this material with $0 \leq x \leq 0.15$. Later D. R. Harshman et al.15 interpreted it as the phase separation into an antiferromagnetic ordered phase and a superconducting phase as in $\mathrm{La}_{2} \mathrm{CuO}_{4+\delta}$ discussed above. J. H. Cho et al 16 measured the spin dynamics of this material with $0.02 \leq x \leq 0.08$ by the ${ }^{139} \mathrm{La}$ nuclear quadrupole resonance (NQR) spin-lattice relaxation rates (NSLR) experiments in 1992. They concluded that the doped holes were inhomogeneously distributed mesoscopically and segregated into walls separating the hole-poor antiferromagnetic domains with size $\approx a / \sqrt{x} \approx$ $10-100 \AA$. Later $\mu^{+}$SR and NQR experiments also support this conclusion 17.18 . Recent NQR experiment shows that in single crystal $\mathrm{La}_{2} \mathrm{CuO}_{4.02}$ there are three different 
regions with different oxygen concentration 19 .

The measurements of the superconducting diamagnetic moments for underdoped and overdoped $\mathrm{La}_{2-x} \mathrm{Sr}_{x} \mathrm{CuO}_{4}$ single crystals show that the underdoped sample has only one transition corresponding to $H_{c 2}$, and the overdoped one has two transitions with the higher one at $H_{c 2}$. The authors proposed that in the two-step transition comes from the phase separation in the overdoped regime 20 .

Several groups have studied the issue of PS by analytical or numerical methods. In the low electron density region, the theoretical results studied by different methods are consistent. Hellberg et al. determined very accurately that the critical $J / t$ for phase separation at low electron density limit is $J / t=3.4367$

But the theoretical results in the low-doping and small $J / t$ region are still conflicting. Emery et al. ${ }^{\mathbb{1}}$ used the exact diagonalization (ED) to study the $4 \times 4$ cluster. The Maxwell construction leads to the conclusion that PS occurs for all values of $J / t$. Hellberg and Manousakis (HM)22. 23 investigated this problem by the Green Function Monte Carlo (GFMC) method and Maxwell construction for larger clusters and reached the similar conclusion that the $t-J$ model phase separates for all values of $J / t$ in the low doping regime. The $U(1)$ slave-boson functional integral results reach the conclusion that for $0<J / t<0.2$ PS occurs for hole density between 0 and 0.124 .

However, the contradictory results are also claimed by several groups. Quantum Monte Carlo (QMC) 256 and ED27 results on the Hubbard model, which should be consistent with the $t-J$ model for small $J / t$, didn't give PS signal. Putikka et al. studied this problem using the high-temperature series expansion and found that at zero temperature, $\mathrm{PS}$ dpes not occur for $\mathrm{J} / t<1.2$ at any electron density 28 22. Prelovšek et al. study the two-point and four-point density correlations on clusters of size 18 and 20 sites. They found that for $J / t>1.5$ the holes form domain walls along $(1,0)$ or $(0,1)$ direction, and phase separate into a hole-rich and a holefree phase for even larger $J / t>2.5$. Poilblanc calculated the energy of 2 and 4 holes by ED on several clusters up to 26 sites. The phase diagram includes a d-wave holepairing state for $J / t \geq 0.2$, a liquid of four-hole droplets (quartets) for larger $J / t \geq 0.5$, and at even larger $J / t$, an instability towards PSBO. Yokoyama et al. investigated the phase diagram by the variational Monte Carlo (VMC) method 31. The critical $J / t$ for phase separation at the high density limit they found is 1.5 , which is consistent with Putikka et al.

The theoretical results of different groups discussed above are consistent at the large $J / t$ and low electron density region. But in the physical regime of high $\mathrm{T}_{c}$ superconductors, $0.3<J / t<0.5$ and high electron density $0.75<n_{e}<0.95$, they are conflicting with each other. We have used the power-Lanczos (PL) method 32 33 to obtain the best estimate of the ground state energy in this physical regime for the largest cluster (122 sites) that have been studied so far. The same lattices and boundary conditions as those used by HME3 are studied also in this report. It can be shown that our data calculated by the PL method, which is a rigorous upper bound of the ground state energies, are still lower than extrapolated GFMC data reported by HM in the physically interested regime. Based on the variational argument we show that there is no phase separation in this physical regime 44 . In this report, we will focus on the competition of the uniform state and PS state. The stripe phase is not taken into account.

\section{NUMERICAL METHOD}

The numerical method used here is the "power-Lanczos method", which is a revised version of Green Function Monte Carlo (GFMC) method 35 , and improved by the Lanczos iterations such that the ground state properties can be calculated more exactly.

The trial wave functions we used in this report is the d-wave RVB (resonating valence bond) with AF correlation 36 ,

$$
\left|\Psi^{T}\right\rangle=\exp \left(h S_{M}\right) P_{d} \prod_{k}\left(u_{k}+v_{k} c_{k \uparrow}^{\dagger} c_{-k \downarrow}^{\dagger}|0\rangle\right.
$$

with

$$
\begin{aligned}
& u_{k}^{2}=\frac{1}{2}\left(1-\frac{\epsilon_{k}}{E_{k}}\right), v_{k}^{2}=\frac{1}{2}\left(1+\frac{\epsilon_{k}}{E_{k}}\right) \\
& \epsilon_{k}=-2\left(\cos _{x}+\cos _{y}\right)-\mu, E_{k}=\sqrt{\left(\epsilon_{k}^{2}+\Delta_{k}^{2}\right)}
\end{aligned}
$$

where $\Delta_{k}$ and $h$ are variational parameters controlling the magnitude of superconductivity and antiferromagnetism. $S_{M}=\sum_{i}(-1)^{x_{i}+y_{i}} S_{z}^{i}$, which is the staggered magnetization. $P_{d}$ operator excludes the states with doubly occupied sites from the wave function to satisfy the constraint of Eq.(2). By tuning the two parameters, the variational energy can be minimized and the trial wave function corresponding to the best set of parameters will be the starting point of the power-Lanczos projection.

The "power method" is the simplified Green function Monte Carlo (GFMC) method, which projects the trial wave function determined variationally toward the true ground state wave function of the Hamiltonian systematically.

Suppose the optimal trial wave function is $\left|\Psi^{T}\right\rangle$. The eigenstates of Hamiltonian are $\left|\Psi^{i}\right\rangle$ and $E_{i}$ are the corresponding eigenenergies. $\left|\Psi^{0}\right\rangle$ denotes the ground state wave function. $\left\{\left|\Psi^{i}\right\rangle\right\}$ form a complete set of wave functions and $\left|\Psi^{T}\right\rangle$ can be expanded as

$$
\left|\Psi^{T}\right\rangle=\sum_{i} a_{i}\left|\Psi^{i}\right\rangle
$$

Applying the operator $(W-H)^{p}$ on $\left|\Psi^{T}\right\rangle$, we get 


$$
\begin{aligned}
\left|\Psi^{(p)}\right\rangle & =(W-H)^{p}\left|\Psi^{T}\right\rangle=\sum_{i}\left(W-E_{i}\right)^{p} a_{i}\left|\Psi^{i}\right\rangle \\
& =\left(W-E_{0}\right)^{p} \sum_{i}\left(\frac{W-E_{i}}{W-E_{0}}\right)^{p} a_{i}\left|\Psi^{i}\right\rangle
\end{aligned}
$$

where $W$ is some constant. If $W$ is properly chosen such that

$$
\left|W-E_{0}\right|>\left|W-E_{i}\right|
$$

for all $i \neq 0$, the weight of excited states will become smaller and smaller when the power $p$ is increasing. In the $p \rightarrow \infty$ limit, all $\left(\frac{W-E_{i \neq 0}}{W-E_{0}}\right)^{p}$ become zero and $\left|\Psi^{(p)}\right\rangle \rightarrow\left|\Psi^{0}\right\rangle$. The trial wave function is projected to the ground state by applying infinite powers of $(W-H)$. For the $t-J$ model, Eq.(7) can be satisfied by setting $W=0$. If $\left|\Psi^{T}\right\rangle$ has the same quantum numbers (S, $S^{z}, \mathbf{k}$, etc.) as the ground state $\left|\Psi^{0}\right\rangle$, and $\left|\Psi^{T}\right\rangle$ is not orthogonal to $\left|\Psi^{0}\right\rangle$, i.e., $a_{0} \neq 0,\left|\Psi^{(p)}\right\rangle=H^{p}\left|\Psi^{T}\right\rangle$ will approach to the ground state as $p \rightarrow \infty$. For a physical quantity $O$ the expectation value of power $p$ is

$$
\begin{array}{r}
\left\langle\Psi^{(p)}|O| \Psi^{(p)}\right\rangle /\left\langle\Psi^{(p)} \mid \Psi^{(p)}\right\rangle= \\
\left\langle\Psi^{T}\left|H^{p} O H^{p}\right| \Psi^{T}\right\rangle /\left\langle\Psi^{T}\left|H^{2 p}\right| \Psi^{T}\right\rangle
\end{array}
$$

Theoretically we can always get the ground state by the power method if we have the trial wave function with correct quantum numbers. However, there are two difficulties with this method. First, the number of intermediate states grow exponentially with powers. Second, the statistical error bar grows exponentially with the applied power due to the famous 'sign' problem of the Fermionic system. We will discuss these two problems below.

A configuration $|a\rangle$ in real space is defined as

$$
|a\rangle=\prod_{i=1}^{N_{e} / 2} c_{r_{i} \uparrow}^{\dagger} \prod_{j=1}^{N_{e} / 2} c_{r_{j} \downarrow}^{\dagger}|0\rangle
$$

where $r_{i}$ and $r_{j}$ represent the positions of the ith up and jth down spins, respectively. $|a\rangle$ satisfies

$$
H|a\rangle=\sum_{a^{\prime}}\left|a^{\prime}\right\rangle\left\langle a^{\prime}|H| a\right\rangle
$$

From Eq.(11), we see that there are three kinds of $\left|a^{\prime}\right\rangle$ such that the coefficient $\left\langle a^{\prime}|H| a\right\rangle \neq 0$ : flipping a nearest-neighbor pair of spin up and down, moving an electron from an occupied site to an empty site, and $|a\rangle$ itself. The number of such $\left|a^{\prime}\right\rangle$ is of order $N_{e}$, the number of electrons. When we apply the next $H$ on it, $H\left|a^{\prime}\right\rangle$ will become a summation of about order $N_{e}$ terms again. To measure the expectation value of some physical quantity $O$ to the $p$ th power, there are $\sim N_{e}^{2 p}$ terms to be calculated. So it is impossible to calculate physical quantities for large power $p$. To avoid this difficulty, we will not sum all the $O\left(N_{e}^{2 p}\right)$ intermediate states for the chosen configurations. Only the important part of them are taken into account by using Monte Carlo method.
The first step is to generate the sequence of $N_{\text {total }}$ configurations $\left|a_{i}\right\rangle, 1 \leq i \leq N_{\text {total }}$, by Metropolis algorithm which is distributed as the weight $P(i)$ corresponding to the trial wave function optimized by VMC method 37 . Our task now is to calculate the matrix elements

$$
\begin{aligned}
\left\langle O^{(p)}\right\rangle_{0} & =\frac{\left\langle\Psi^{T}\left|H^{p} O H^{p}\right| \Psi^{T}\right\rangle}{\left\langle\Psi^{T} \mid \Psi^{T}\right\rangle}=\sum_{i} P(i) O^{(p)}(i) \\
P(i) & =\frac{\left|C_{i}\right|^{2}}{\sum_{i^{\prime}}\left|C_{i^{\prime}}\right|^{2}} \\
O^{(p)}(i) & =\frac{\sum_{i^{\prime}}\left\langle a_{i}\left|H^{p} O H^{p}\right| a_{i^{\prime}}\right\rangle C_{i^{\prime}}}{C_{i}}
\end{aligned}
$$

where $C_{i}$ is the amplitude of the configuration $\left|a_{i}\right\rangle$ of the trial wave function $\left.\Psi^{T}\right\rangle$. The operator $O$ equals to the Hamiltonian operator $H$ when we measure the energy. The first task is to calculate $\left\langle H^{n}\right\rangle$ which will be useful for calculating energy $(n=2 p+1)$, and normalization factor $(n=2 p)$. Expanding the matrix element in the following form

$$
\begin{aligned}
\left\langle a_{i}\left|H^{n}\right| a_{f}\right\rangle= & \sum_{m_{1}, m_{2}, \ldots, m_{n-1}}\left\langle a_{i}|H| m_{1}\right\rangle\left\langle m_{1} H \mid m_{2}\right\rangle \ldots \\
& \left\langle m_{n-2}|H| m_{n-1}\right\rangle\left\langle m_{n-1}|H| a_{f}\right\rangle \quad \text { (12) }
\end{aligned}
$$

Define

$$
P_{\alpha \beta}=P_{|\alpha\rangle \rightarrow|\beta\rangle}=\frac{1}{Z_{\alpha}} H_{\alpha \beta}
$$

with $Z_{\alpha}=\sum_{\beta} H_{\alpha \beta}$ and $H_{\alpha \beta}=\langle\alpha|H| \beta\rangle$. Eq.(12) can be rewritten as

$$
\begin{aligned}
&\left\langle a_{i}\left|H^{n}\right| a_{f}\right\rangle= \sum_{m_{1}, m_{2}, \ldots, m_{n-1}}\left(P_{a_{i} m_{1}} Z_{a_{i}}\right)\left(P_{m_{1} m_{2}} Z_{m_{1}}\right) \ldots \\
&\left(P_{m_{n-1} a_{f}} Z_{m_{n-1}}\right)
\end{aligned}
$$

To calculate this exactly all the possible paths $\left\{m_{1}, m_{2}, \ldots, m_{n-1}\right\}$ connecting the initial and final configuration $\left|a_{i}\right\rangle$ and $\left|a_{f}\right\rangle$ have to be taken into account. Since there are about $N_{e}^{n}$ terms, it is impossible to sum all the terms. Instead we make use of the random walk procedure.

Starting from the initial configuration $\left|a_{i}\right\rangle$, use $P_{a_{i} m_{1}^{\prime}}$ defined in Eq.(13) to be the probability of transition from $\left|a_{i}\right\rangle$ to $\left|m_{1}^{\prime}\right\rangle$ state. There are many possible $\left|m_{i}^{\prime}\right\rangle$ of three types corresponding to nearest neighbor spinflipping, hole-hopping, and diagonal terms as mentioned earlier in this section. For these types the corresponding matrix elements $H_{a_{i} m_{1}^{\prime}}$ are $-t,-J / 2$, and $-J / 2$, respectively. Choose one of these $\left|m_{1}^{\prime}\right\rangle$ to be the $\left|m_{1}\right\rangle$ randomly with the transition probability $P_{a_{i} m_{1}^{\prime}}$. Then use the same procedure to generate the next intermediate state $\left|m_{2}\right\rangle$. Repeat this $n$ times, we can arrive the final state $\left|a_{f}\right\rangle$ of this path. The measurement of $\left\langle H^{n}\right\rangle$ for this path is

$$
M_{i}=\left(Z_{a_{i}} \cdot Z_{m_{1}} \cdot Z_{m_{2}} \cdots Z_{m_{n-1}}\right) C_{a_{f}}^{T} / C_{a_{i}}^{T}
$$


This is only one term in the summation of Eq.(14). Of course the error will be large if we use this value to estimate the summation in Eq.(14). To reduce the error, we generate many paths by the procedure above and average the measurement

$$
\bar{M}_{i}=\frac{1}{N_{\text {path }}} \sum_{k=1}^{N_{\text {path }}} Z_{a_{i}} Z_{m_{1}^{k}} Z_{m_{2}^{k}} Z_{m_{3}^{k}} \cdots Z_{m_{n-1}^{k}} \cdot \frac{C_{a_{f}^{k}}^{T}}{C_{a_{i}}^{T}}
$$

$N_{\text {path }}$ is total number of paths of random walk with the same initial configuration $\left.\left|a_{i}\right\rangle . \quad\left\{\left|m_{1}^{k}\right\rangle,\left|m_{2}^{k}, \ldots,\right| a_{f}^{k}\right\rangle\right\}$ is the intermediate and final states of the $k$ th path. $N_{\text {path }}$ should be large enough to get good statistics. Typical $N_{\text {path }}$ is of order $10^{2} \sim 10^{3}$. Together with Eq.(11), and Eq.(16) we get

$$
\left\langle H^{n}\right\rangle=\langle M\rangle=\frac{1}{N_{\text {total }}} \sum_{i=1}^{N_{\text {total }}} \bar{M}_{i} \pm \delta
$$

with

$$
\begin{aligned}
\delta & =\sqrt{\frac{1}{N_{\text {total }}}\left(\left\langle M^{2}\right\rangle-\langle M\rangle^{2}\right)} \\
\left\langle M^{2}\right\rangle & =\frac{1}{N_{\text {total }}} \sum_{i=1}^{N_{\text {total }}} \bar{M}_{i}^{2}
\end{aligned}
$$

Now we can calculate $\left\langle H^{n}\right\rangle$. With this it is straightforward to calculate the energy of $p$ th power $E^{(p)}=$ $\left\langle H^{2 p+1}\right\rangle /\left\langle H^{2 p}\right\rangle$.

Another difficulty is the 'fermionic sign problem'. The matrix elements of Hamiltionian $\langle\alpha|H| \beta\rangle$ are not positive or negative definite. That is, some of the weight Eq.(15) of the $N_{\text {path }}$ paths are positive and others are negative. The summation in Eq.(16), or Eq.(17) will become a summation of a positive part and a negative part

$$
\left\langle H^{p} O H^{p}\right\rangle=\left(O_{+}^{(p)}+O_{-}^{(p)}\right) \pm\left(\sigma_{+}^{(p)}+\sigma_{-}^{(p)}\right)
$$

where $O_{+}^{(p)}$ and $O_{-}^{(p)}$ are the positive and negative parts of the summation in Eq. 177).

For smaller powers, the portion of positive part is much larger than the negative part. It is because that a step of random walk only flips a pair of nearest neighbor spins or moves an electron to the nearest neighbor empty site, and the probability of crossing the nodes of the trial wave function and resulting a negative matrix element is small. For large powers, the probability of crossing the nodes of the trial wave function will be accumulated during the long path of random walk and the negative part in the summation Eq.(16) and Eq.(17) will grow larger. The larger the negative portion (absolute value closer to the positive portion) the larger the error bars of the physical measurements will be. In Fig.1(a) we show the ratio of the negative and positive portions varies with power for $J / t=0.4$ and electron density $n_{e}=10 / 16,10 / 36,18 / 36$, and $26 / 36$. It is clear that the negative portion is larger for higher electron density. For the high density cases $10 / 16$ and $26 / 36$, the absolute value of negative part is almost the same as the positive part for $\left\langle H^{20}\right\rangle$. Thus | $\left\langle H^{20}\right\rangle|\ll|\left\langle H^{20}\right\rangle_{ \pm} \mid$and the statistical error of $\left\langle H^{20}\right\rangle$ will be largely increased. For example, the negative ratio is about $79 \%$ for $10 / 36,87 \%$ for $18 / 36$, and $97 \%$ for $26 / 36$.
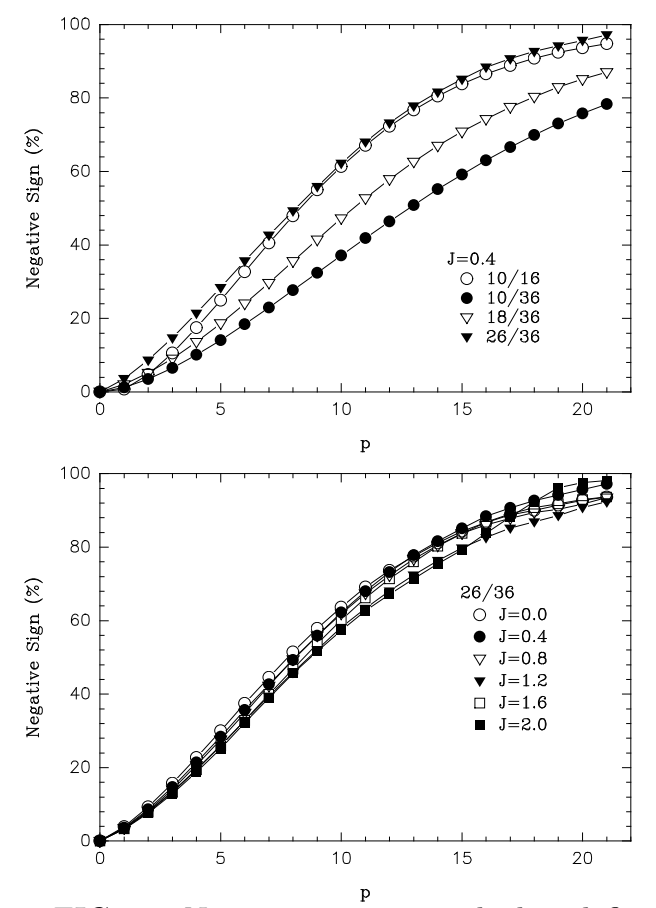

FIG. 1. Negative sign ratio which is defined as the ratio of the absolute value of negative and positive parts of $\left\langle H^{n}\right\rangle$ for (a) different electron densities: 10/16 (open circle), 10/36 (full circle), 18/36 (open triangle) and 26/36 (full triangle) and (b) different $J / t$ values: $J / t=0$ (open circle), 0.4 (full circle), 0.8 (open triangle), 1.2 (full triangle), 1.6 (open square) and 2.0 (full square).

If we choose $N_{\text {total }}$ and $N_{\text {path }}$ large enough to reduce the error bars of $\left\langle H^{20}\right\rangle_{ \pm}$to be as small as $0.1 \%$, the corresponding error bars of $\left\langle H^{20}\right\rangle$ of these three electron densities will be $1 \%, 1.5 \%$, and $6.7 \%$. So no matter how large $N_{\text {total }}$ and $N_{\text {path }}$ are, and how accurate $O_{ \pm}^{(p)}$ are, the sign problem always prevents us to do calculations for large powers, especially in the high electron density regime. The largest power we use is usually 6 or 8 for high electron densities. The negative ratio is about $80 \% \sim 90 \%$. Beyond this the statistical errors are too large to be reliable. The energies varies with power for different densities are plotted in the Fig.2 (open circles) for $J / t=0.4$. In Fig.1(b) the negative ratio for different $J / t$ value of 26/36 system is shown. By comparing Fig.1 (a) and (b) we see the negative ratio depends on the electron density much more sensitive than $J / t$.

The sign problem is the intrinsic limitation of the power method. It is almost impossible to project the trial wave function to be very close to the ground state by applying large powers on it, especially in the physi- 
cal interested region with high electron density. Other methods like QMC or GFMC also encounter a similar problem. To overcome this limitation, we have modi-

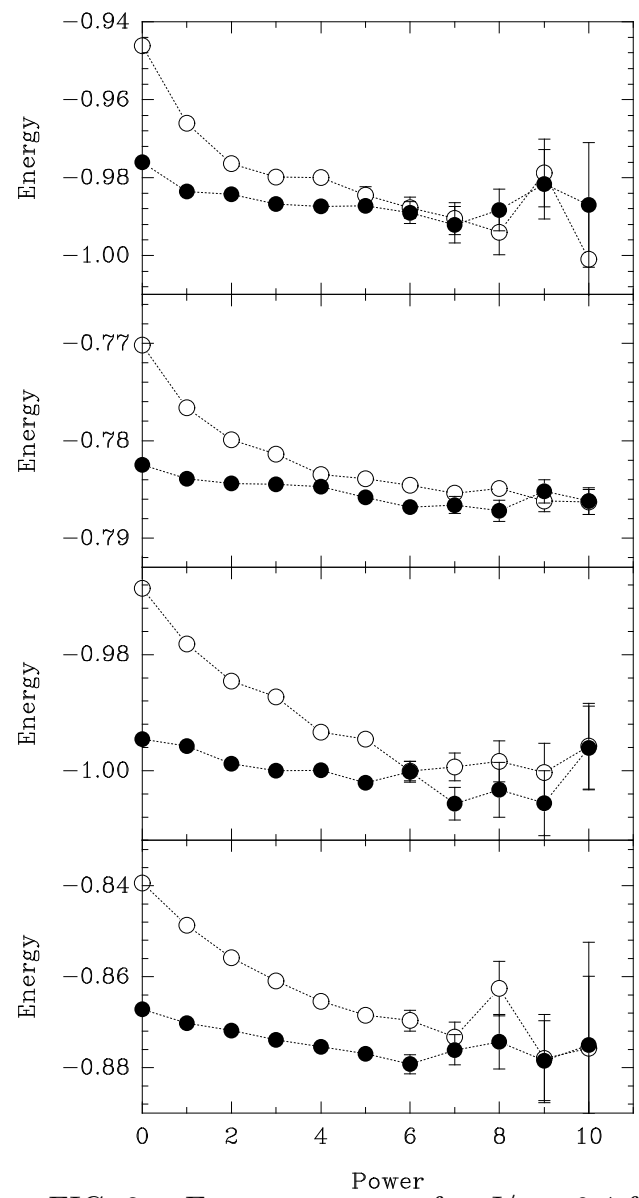

FIG. 2. Energy vs power for $J / t=0.4$ for $10 / 16,10 / 36$, $18 / 36$, and $26 / 36$ (from the top to the bottom, open circles). The full circles are the PL1 data.

fied the power method. Here we will introduce the more powerful power-Lanczos method 32 .

An inappropriately chosen trial wave function would require a lot of computer time to converge to the ground state because it is necessary to apply many powers on the trial wave function. Hence large $N_{\text {total }}$ and $N_{\text {path }}$ will be necessary to reduce the statistical error due to the random walk approximation and the fermionic negative sign problem. Since the negative sign problem prevents us to do large powers to get the results close to the ground state in the physically interested high density region, another solution is to make our starting point, the trial wave function closer to the ground state. If our trial wave function is closer to the ground state, it will not be necessary to apply many powers. And the negative impact of sign problem can be reduced. Here we use the Lanczos algorithm to improve our trial wave function, and then apply powers of Hamiltonian on the new trial wave function. The method is a combination of power method introduced in the previous section and the Lanc- zos iteration method usually used in the exact diagonalization (ED) for the small clusters. Hence it was named as 'power-Lanczos method'. Lanczos iteration method was first proposed by Heeb and Rice to improve the trial wave function systematically 33 . The effectiveness of the method is demonstrated on the two-dimensional antiferromagnetic Heisenberg model and later on the twodimensional $t-J$ model 38 . We will describe their method below.

Starting from a trial wave function $\left|\phi_{0}\right\rangle$ (which we called it $\left|\Psi^{T}\right\rangle$ above) optimized by the variational method. Apply the Hamiltonian $H$ on $\left|\phi_{0}\right\rangle$. Since $\left|\phi_{0}\right\rangle$ is not an eigenstate of $H$, it becomes

$$
H\left|\phi_{0}\right\rangle=a_{0}\left|\phi_{0}\right\rangle+b_{1}\left|\phi_{1}\right\rangle
$$

with

$$
\left\langle\phi_{0} \mid \phi_{1}\right\rangle=0
$$

which means $\left|\phi_{0}\right\rangle$ and $\left|\phi_{1}\right\rangle$ are orthogonal. $a_{0}=\left\langle\phi_{0}\right|$ $H\left|\phi_{0}\right\rangle=E_{0}$, which is the expectation value of energy of the trial wave function. And

$$
\begin{aligned}
b_{1} & =\left\langle\phi_{1}|H| \phi_{0}\right\rangle \\
& =\frac{1}{b_{1}}\left\langle\phi_{0}\left|\left(H-E_{0}\right) H\right| \phi_{0}\right\rangle
\end{aligned}
$$

This formalism is similar to the Lanczos iteration for exact diagonalization, which the recurrence relation is

$$
\begin{aligned}
& H\left|\phi_{0}\right\rangle=a_{0}\left|\phi_{0}\right\rangle+b_{1}\left|\phi_{1}\right\rangle \\
& H\left|\phi_{n}\right\rangle=a_{n}\left|\phi_{n}\right\rangle+b_{n}\left|\phi_{n-1}\right\rangle+b_{n+1}\left|\phi_{n+1}\right\rangle
\end{aligned}
$$

For small systems, $n$ can be as large as the number of all the possible configurations in real space and $H$ can be diagonalized exactly in the basis $\left\{\left|\phi_{0}\right\rangle,\left|\phi_{1}\right\rangle, \ldots,\left|\phi_{n}\right\rangle\right\}$ and the eigenstate corresponding the lowest eigenenergy is just the ground state we need. The largest cluster solved by this method exactly is 32 sites 39 . Although for larger clusters it is impossible to use this method to solve the exact ground state, we can truncate the recurrence relation Eq.(23) at $\mathrm{n}$ which is small enough such that we can calculate at large systems, and then diagonalize the Hamiltonian by the incomplete basis. The lowestenergy eigenstate will not be the ground state because the basis is incomplete but it will be much closer to the ground state than the original trial wave function even $n$ is small.

Now consider the first order iteration $(n=1)$ which we call it 'PL1' in Eq. (23)

$$
\begin{aligned}
& H\left|\phi_{0}\right\rangle=a_{0}\left|\phi_{0}\right\rangle+b_{1}\left|\phi_{1}\right\rangle \\
& H\left|\phi_{1}\right\rangle=a_{1}\left|\phi_{1}\right\rangle+b_{1}\left|\phi_{0}\right\rangle+b_{2}\left|\phi_{2}\right\rangle
\end{aligned}
$$

where $\left\langle\phi_{2} \mid \phi_{0}\right\rangle=0$ and $\left\langle\phi_{2} \mid \phi_{1}\right\rangle=0$. Since we truncate the recurrence relation Eq. 23) at $n=1$ here, the last term in Eq.(24) is dropped and the Hamiltonian can be 
written in the space spanned by the basis $\left\{\left|\phi_{0}\right\rangle,\left|\phi_{1}\right\rangle\right\}$ as

$$
H=\left(\begin{array}{cc}
H_{00} & H_{01} \\
H_{10} & H_{11}
\end{array}\right)=\left(\begin{array}{cc}
E_{0} & b_{1} \\
b_{1} & a_{1}
\end{array}\right)
$$

where $E_{0}=a_{0}$. Remember that this is the first order approximation because we drop the second order term in Eq.(24). Under this approximation, $a_{1}$ in Eq.(24) is

$$
\begin{aligned}
a_{1} & =\left\langle\phi_{1}|H| \phi_{1}\right\rangle \\
& =\frac{1}{b_{1}^{2}}\left\langle\phi_{0}\left|\left(H-E_{0}\right) H\left(H-E_{0}\right)\right| \phi_{0}\right\rangle \\
& =\frac{\left\langle\phi_{0}\left|\left(H-E_{0}\right) H\left(H-E_{0}\right)\right| \phi_{0}\right\rangle}{\left\langle\phi_{0}\left|\left(H-E_{0}\right) H\right| \phi_{0}\right\rangle}
\end{aligned}
$$

Diagonalize the Hamiltonian in Eq.(25), the smaller eigenvalue and the corresponding eigenstate is

$$
\begin{aligned}
E_{1} & =\frac{1}{2}\left[E_{0}+a_{1}-\sqrt{\left(E_{0}-a_{1}\right)^{2}+b_{1}^{2}}\right] \\
|P L 1\rangle & =\left|\phi_{0}\right\rangle+\frac{b_{1}}{E_{1}-a_{1}}\left|\phi_{1}\right\rangle
\end{aligned}
$$

We have to know the weight of each configuration in real space of the new trial wave function $|P L 1\rangle$. If

$$
|P L 0\rangle=\left|\phi_{0}\right\rangle=\sum_{i} a_{i}^{(0)}|i\rangle
$$

and

$$
|P L 1\rangle=\sum_{i} a_{i}^{(1)}|i\rangle
$$

where $a_{i}^{(0)}$ is the amplitude of $|i\rangle$. Then

$$
\begin{aligned}
a_{i}^{(1)} & =\langle i \mid P L 1\rangle \\
& =\left\langlei \left|\left(|P L 0\rangle+\frac{b_{1}}{E_{1}-a_{1}}\left|\phi_{1}\right\rangle\right)\right.\right. \\
& =a_{i}^{0}+\frac{b_{1}}{E_{1}-a_{1}}\left\langle i \mid \phi_{1}\right\rangle
\end{aligned}
$$

From Eq.(20) we know $b_{1}\left|\phi_{1}\right\rangle=\left(H-E_{0}\right)|P L 0\rangle$. Thus

$$
\begin{aligned}
a_{i}^{(1)} & =a_{i}^{(0)}+\frac{1}{E_{1}-a_{1}}\left\langle i\left|\left(H-E_{0}\right)\right| P L 0\right\rangle \\
& =a_{i}^{(0)}\left(1-\frac{E_{0}}{E_{1}-a_{1}}\right)+\frac{1}{E_{1}-a_{1}} \sum_{j}\langle i|H| j\rangle a_{j}^{(0)}
\end{aligned}
$$

Thus the ratio of the weight of $|P L 1\rangle$ and $|P L 0\rangle$ corresponding the same configuration $|i\rangle$ is

$$
\frac{a_{i}^{(1)}}{a_{i}^{(0)}}=1-\frac{E_{0}}{E_{1}-a_{1}}+\frac{1}{E_{1}-a_{1}} \sum_{j}\langle i|H| j\rangle \frac{a_{j}^{(0)}}{a_{i}^{(0)}}
$$

Heeb and Rice 33 proposed to calculate $a_{1}$ and $b_{1}$ by using Monte Carlo technique. With $a_{1}$ and $b_{1}, E_{1}$ and
$|P L 1\rangle$ can be calculated from Eq.(27). However, in this method $a_{1}$ and $b_{1}$ have to be calculated very accurately. A small error will produce large uncertainty in $E_{1}$ and $|P L 1\rangle$. The statistical error is inevitable since they are calculated by Monte Carlo method. Here we choose an alternative. Since $|P L 1\rangle$ in Eq.(27) has not been properly normalized. All the $a_{i}^{(1)}$ can be divided by a constant $\left(1-\frac{E_{0}}{E_{1}-a_{1}}\right)$ and Eq.(30) becomes

$$
\frac{a_{i}^{(1)}}{a_{i}^{(0)}}=1+C_{1} \sum_{j}\langle i|H| j\rangle \frac{a_{j}^{(0)}}{a_{i}^{(0)}}
$$

with

$$
C_{1}=\frac{1}{E_{1}-a_{1}-E_{0}}
$$

Note that $C_{1}$ is a constant once the trial wave function $|P L 0\rangle=\left|\phi_{0}\right\rangle$ is chosen. Instead of calculating $C_{1}$ by using $a_{1}$ and $b_{1}$ obtained by Monte Carlo method, we treat $C_{1}$ as a variational parameter to optimize the energy. The optimized energy is just $E_{1}$. For each configuration the PL0 weight of the trial wave function has to be calculated first and the PL1 weight can be evaluated by using Eq.(31). This procedure largely increases the computer time (approximately of order $N_{e}$ ). To sawe computer time Ceperley's algorithm for ratio of weight 37 is used for calculating $a_{j}^{(0)} / a_{i}^{(0)}$ where $|i\rangle$ and $|j\rangle$ are connected by $H$. It is important to check the ratio of determinant to prevent the divergence of the ratio. $a_{j}^{(0)}$ and $a_{i}^{(0)}$ should be calculated directly if their ratio obtained by Ceperley's method is too large or too small, for example, larger than $10^{8}$ or less than $10^{-8}$. Otherwise the results evaluated by using Ceperley's method might be in err.

To estimate the possible range of $C_{1}=1 /\left(E_{1}-a_{1}-\right.$ $E_{0}$ ), from Eq.(26) it is easy to show

$$
a_{1}=\frac{\left\langle\phi_{0}\left|\left(H-E_{0}\right)^{3}\right| \phi_{0}\right\rangle}{\left\langle\phi_{0}\left|\left(H-E_{0}\right)^{2}\right| \phi_{0}\right\rangle}+E_{0}
$$

If our trial wave function is not too bad, roughly we can estimate that $E_{1} \approx E_{0}$ and $a_{1} \approx E_{0}$. So the range we tuning $C_{1}$ is around $-1 / E_{0}$. Typical behavior of $E_{1}$ vs $C_{1}$ is shown in Fig.3.

From our experience of PL1 calculation, the $\mathrm{PL} 1_{\text {power }=0}$ energy $E_{1}$ is approximately equal to the energy of $\mathrm{PL} 0_{\text {power }}=4$ or $\mathrm{PL} 0_{\text {power }}=5$. Our new trial wave function $|P L 1\rangle$ is much better than the original trial wave function $|P L 0\rangle$. The next step is to improve the wave function further. There are two ways to achieve this purpose: (1) using power method introduced in the previous section, applying powers on $|P L 1\rangle$ and projecting it to the ground state systematically; (2) using the next order of Lanczos iteration, i.e., the 'PL2'.

First let's see the first method: 'PL1-power' calculation. We can use the same procedure introduced previously to apply $H^{p}$ on the new wave function $|P L 1\rangle$. 


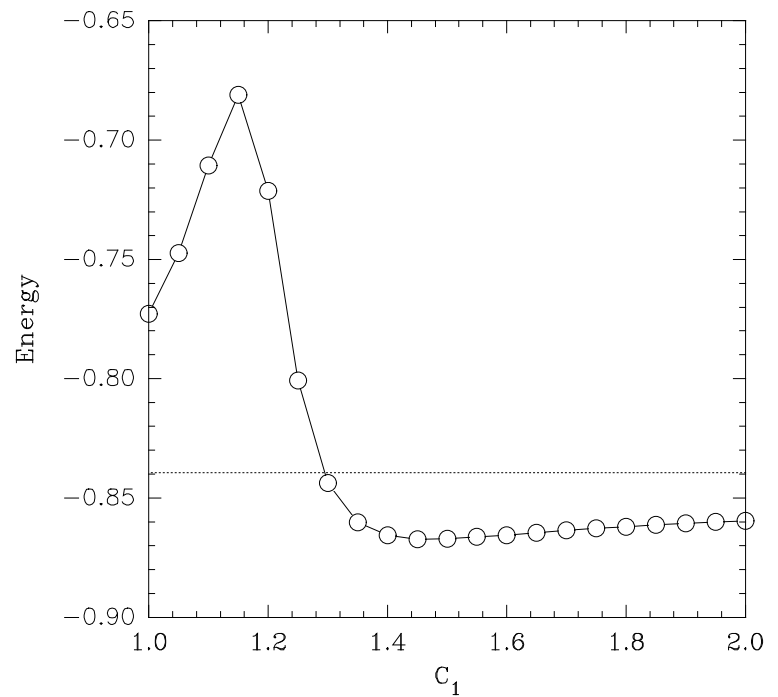

FIG. 3. PL1 $1_{\text {power }=0}$ energy dependence of $C_{1}$ for $26 / 36$, $J / t=0.4$. the horizontal dotted line represents the energy of $\mathrm{PLO}_{\text {power }}=0$.

The only difference is that the weight of the configurations has to be changed from PL0 to PL1 (Eq.(31)). As in the PL0-power calculation, the PL1-power calculation is suffered from the fermionic sign problem. In Fig.4 the negative sign ratio vs power for $|P L 1\rangle$ is shown for $J / t=0.4$ in $10 / 16,10 / 36,18 / 36$, and $26 / 36$ lattices. It is clear that the behavior of negative sign ratio vs power for $|P L 0\rangle$ and $|P L 1\rangle$ are almost the same, which means the maximum power under the limitation of negative sign problem is the same for both $|P L 0\rangle$ and $|P L 1\rangle$. Since $|P L 1\rangle$ is closer to the ground state than $|P L 0\rangle$, the best PL1-power energy will be lower than that of PL0. Fig. 2 shows the PL0 and PL1-power energy for $J / t=0.4$ in $10 / 16,10 / 36,18 / 36$, and $26 / 36$ sites (full circles). It is clear that PL1 always yields lower energies.

Another way to improve the wave function further is to do PL2. Since we have the equations for $|P L 1\rangle$, we can follow the same procedure which evaluates $|P L 1\rangle$ from $|P L 0\rangle$ to generate $|P L 2\rangle$ from $|P L 1\rangle$. For a configuration $|i\rangle$ the weight corresponding $|P L 2\rangle$ is

$$
a_{i}^{(2)}=a_{i}^{(1)}+C_{2} \sum_{j}\langle i|H| j\rangle a_{j}^{(1)}
$$

which is completely in the same form as Eq.(31).

Using Eq.(31) to replace $a_{i}^{(1)}$ by the expression of $a_{i}^{(0)}$ we get

$$
\begin{aligned}
a_{i}^{(2)} & =a_{i}^{(0)}+C_{1} \sum_{k}\langle i|H| k\rangle a_{k}^{(0)} \\
& +C_{2} \sum_{j}\langle i|H| j\rangle\left(a_{j}^{(0)}+C_{1} \sum_{k}\langle j|H| k\rangle a_{k}^{(0)}\right) \\
\frac{a_{i}^{(2)}}{a_{i}^{(0)}} & =1+\left(C_{1}+C_{2}\right) \sum_{k}\langle i|H| k\rangle \frac{a_{k}^{(0)}}{a_{i}^{(0)}}
\end{aligned}
$$

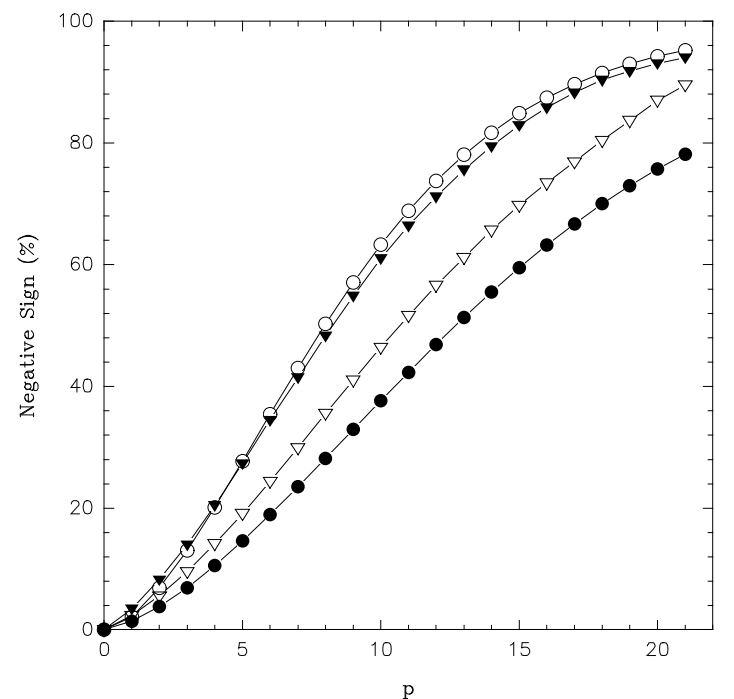

FIG. 4. PL1 negative sign ratio which is defined as the ratio of the absolute value of negative and positive parts of $\left\langle H^{n}\right\rangle$ for different electron densities: 10/16 (open circle), 10/36 (full circle), 18/36 (open triangle) and 26/36 (full triangle).

$$
+C_{1} C_{2} \sum_{j k}\langle i|H| j\rangle\langle j|H| k\rangle \frac{a_{k}^{(0)}}{a_{i}^{(0)}}
$$

The $|P L 2\rangle$ will be closer to the ground state than $|P L 1\rangle$, and in principle we can apply powers of $H$ on $|P L 2\rangle$ to project it toward the ground state. The PL2-power results should be better than that of PL1-power. With the similar rough estimation as for PL1 $C_{1}, C_{1}$ and $C_{2}$ in Eq.(35) are near the value $1 / E_{0}$. Chen and Lee 32 calculated PL2 in 16 sites. The PL2 energy is very close to the ground state energy. In practice, for larger lattices, it is quite difficult to find the optimal values of $C_{1}$ and $C_{2}$ by using the VMC method because the behavior of variational energy in the two-dimensional parameter space $\left\{C_{1}, C_{2}\right\}$ is much more complicated than the 1parameter case in PL1 calculation. Another problem is that the PL2 calculation costs much more computer time than PL0 and PL1. We are searching for ways to carry out the PL2 calculation more efficiently. In this paper, most data are evaluated from the PL0 and PL1 calculations.

Note that the energy calculated from the powerLanczos method is still a variational estimate and an upper bound of the ground state energy.

Here we demonstrate an example of the energies calculated by the PL method, and compare that with some recent data by other groups. For 50 electrons in an $8 \times 8$ lattice and $J / t=0.2$, the PL energies are shown in TABLE四.

It can be seen from TABLE II that the PL1 and PL2 energies converge much faster than PL0 (GFMC) ones. The upper bound of the ground state energy can be estimated with less powers such that the fermionic sign problem is also reduced significantly.

Another interesting point is the ratio of spin $\left(E_{s}\right)$ and 
kinetic $\left(E_{k}\right)$ energies. Fig. 5 shows the two portions of energy of different powers for $50 / 64, J / t=0.2$. It can be seen that $E_{s}$ is overestimated while $E_{k}$ is underestimated in the trial wave function. From our experiences, $E_{s}$ is usually overestimated for the sake of minimizing the energy of the trial wave function in the physical interested region of the parameter space. The choice causes misjudgements of other physical quantities. For example, the superconducting long range pairing correlation function will be strongly overestimated if we choose the energy-optimized RVB wave function 40 . From the behavior of $E_{s}$ and $E_{k}$, we see that it is a very important task to find a new trial wave function having more reasonable $E_{s} / E_{k}$ value, as well as having lower trial energy.

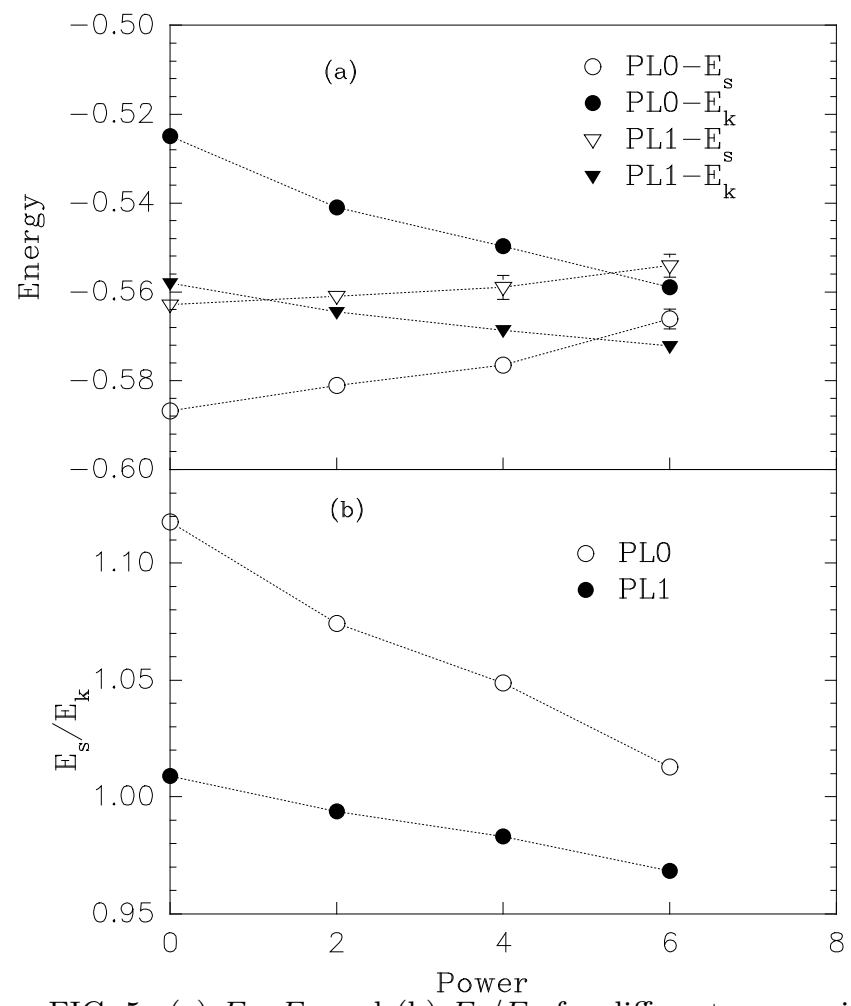

FIG. 5. (a) $E_{s}, E_{k}$ and (b) $E_{s} / E_{k}$ for different powers in $50 / 64, J / t=0.2$.

In conclusion of this section, we proposed a powerful method which is a combination of Lanczos iteration and power method. The Lanczos iteration is used to improve the starting point of the power method which projects the new $(\mathrm{PL})$ trial wave function toward the ground state. The ground state properties can be estimated more accurately under the limitation of the fermionic sign problem.

\section{EXTRAPOLATION OF THE ENERGIES}

From TABLE I we conclude that the ground state energy of this system is lower than -0.6855 by the variational principle. HM23 gives $-0.6825(23)$ by extrapolating the GFMC data. They used a different method
TABLE I. Power-Lanczos energies of $J / t=0.2$ for 50 electrons in a $8 \times 8$ lattice.

\begin{tabular}{|c|c|c|c|}
\hline \hline power & PL0 & PL1 & PL2 \\
\hline 0 & $-0.6443(1)$ & $-0.6709(1)$ & $-0.6819(11)$ \\
\hline 2 & $-0.6573(4)$ & $-0.6768(2)$ & $-0.6833(11)$ \\
\hline 4 & $-0.6657(6)$ & $-0.6803(6)$ & $-0.6851(29)$ \\
\hline 6 & $-0.6735(9)$ & $-0.6820(8)$ & \\
\hline 8 & $-0.6769(12)$ & $-0.6855(13)$ & \\
\hline \hline
\end{tabular}

to calculate energies of all different powers in the same time. The energies of different powers calculated by HM are close to our PL0-power data. It can be seen that the extrapolated GFMC energy is still higher than the variational upper bound determined by $\mathrm{PL}_{\text {power }=8}$ or $\mathrm{PL} 2_{\text {power }=4}$ from TABLE I. Our energy vs. power curves for PL0 and PL1 are fitted by an exponential function:

$$
E=E_{0}+a e^{-b p}
$$

The results are shown in Fig.6. For PL0 data, the fitting

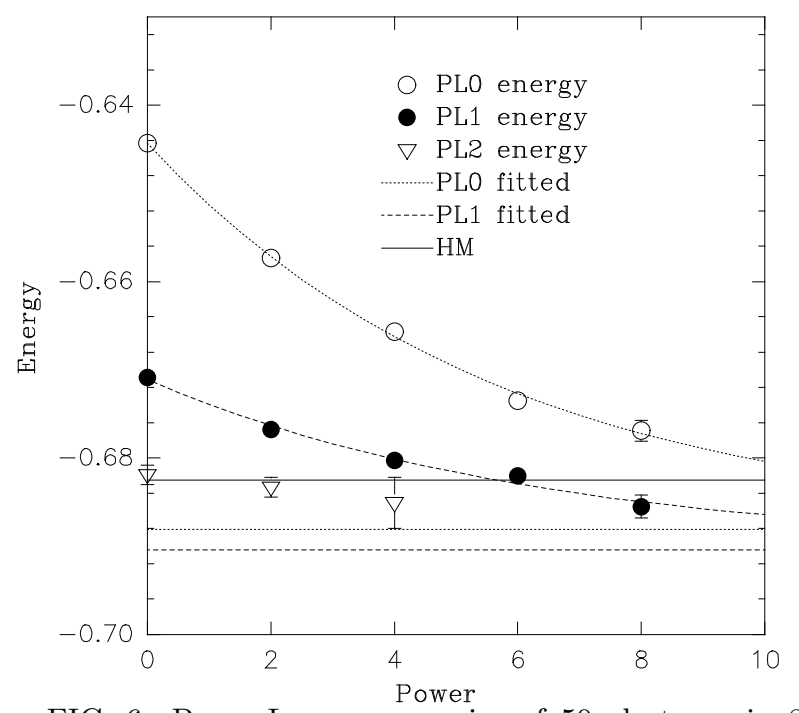

FIG. 6. Power-Lanczos energies of 50 electrons in $8 \times 8$ lattice, $J / t=0.2$. The PL0 (PL1, PL2) data are represented by open circles (full circles, open triangles). The dotted and dashed curves fit the PL0 and PL1 data, respectively, and the horizontal dotted and dashed lines are the extrapolated values of power $=\infty$. The horizontal solid line is the ground state value estimated by HM.

parameters are $E_{0}=-0.6881(35), a=0.0438(33)$, and $a=0.174(26)$. And $E_{0}=-0.6904(48), a=0.0193(46)$, and $b=0.158(70)$ for PL1 energies. The most important parameter is $E_{0}$, the values for PL0 and PL1 data are consistent, which are both much smaller than HM result. Since the ground state energy must be lower than the lowest power-Lanczos energy, their extrapolated data is clearly inaccurate.

In TABLE II, we show $J / t=0.2$ energies for several electron densities. The lattices and boundary conditions 
are chosen exactly the same as those chosen by Hellberg et al.23.

$E_{\text {extrap }}$ are the data extrapolated from GFMC and $E_{\text {lanc }}$ are the quoted values for Lanczos iterations 23 . It can be seen that for this $J / t, E_{P L}$, the PL energies are lower than $E_{\text {extrap }}$ for higher densities, and higher than $E_{\text {extrap }}$ for intermediate ones (50/90 and 50/72).

We emphasize here that in the cases of high density and small coupling constant, it is difficult to get accurate enough energies for powers larger than 8 because the sign problem enlarges the statistical error bar for such large powers. Thus it is unreliable to extrapolate the GFMC data to estimate the ground energy in this regime for larger clusters, even though the method works well in the small clusters like $4 \times 4$, or at low density and large $J / t$ regime because the energies can be calculated very accurately for more powers, and the convergence in these cases is much faster than the high density, small $J / t$ in large clusters. For example, the difference between exact energy and the number evaluated by PL1-power $=10$ for $10 / 16$ is about only $0.1 \%$.

TABLE II. Energies of $J / t=0.2$ for several densities and lattices evaluated by PL method $E_{P L}$, extrapolated GFMC $E_{\text {extrap }}$, and Lanczos iterations $E_{\text {lanc. }}$.

\begin{tabular}{|c|c|c|c|}
\hline \hline$N_{e} / N_{s}$ & $E_{P L}$ & $E_{\text {extrap }}$ & $E_{\text {lanc }}$ \\
\hline $50 / 90$ & $-0.9184(13)$ & $-0.9246(35)$ & $-0.9211(54)$ \\
\hline $50 / 72$ & $-0.8074(11)$ & $-0.8103(28)$ & $-0.8098(32)$ \\
\hline $50 / 64$ & $-0.6855(13)$ & $-0.6825(23)$ & $-0.6826(27)$ \\
\hline $50 / 56$ & $-0.4708(8)$ & $-0.4681(18)$ & $-0.4717(23)$ \\
\hline $60 / 64$ & $-0.3710(6)$ & $-0.3701(17)$ & $-0.3693(7)$ \\
\hline \hline
\end{tabular}

\section{DETERMINATION OF PHASE SEPARATION: MAXWELL CONSTRUCTION}

There are several criteria to determine the boundary of PS. The standard one is to find the density of divergence of the compressibility (the second derivative of the energy-density curve). This method is successful in determining the phase boundary of the one-dimensional $t-J$ model, which phase separates into one electron-free regiop and another one containing both electrons and holes 41 .

For the 2D $t-J$ model, the PS state contains one holefree Heisenberg AF region and another hole-containing one. The finite size effect from the "surface energy" between the hole-free Heisenberg region of the PS state is significant (of the order $1 / \sqrt{N_{s}}$, where $N_{s}$ is the size of the lattice), and this surface energy will push the PS state to much larger $J / t$ value. If we use the inverse compressibility method in the $2 \mathrm{D} t-J$ model, we will get wrong results due to this effect. Thus we have to use the Maxwell construction method to find the PS boundary from the energies of the finite-size, uniform systems.
The energy approaches the ground state as the power increases in our PL method. Although in the physical regime most of our best data have not yet converged to the exact ground state values, the systematic variation of energies is enough for us to give a variational estimate of the lower bound of the phase separation boundary. We will use the lowest PL energies in the following discussion.

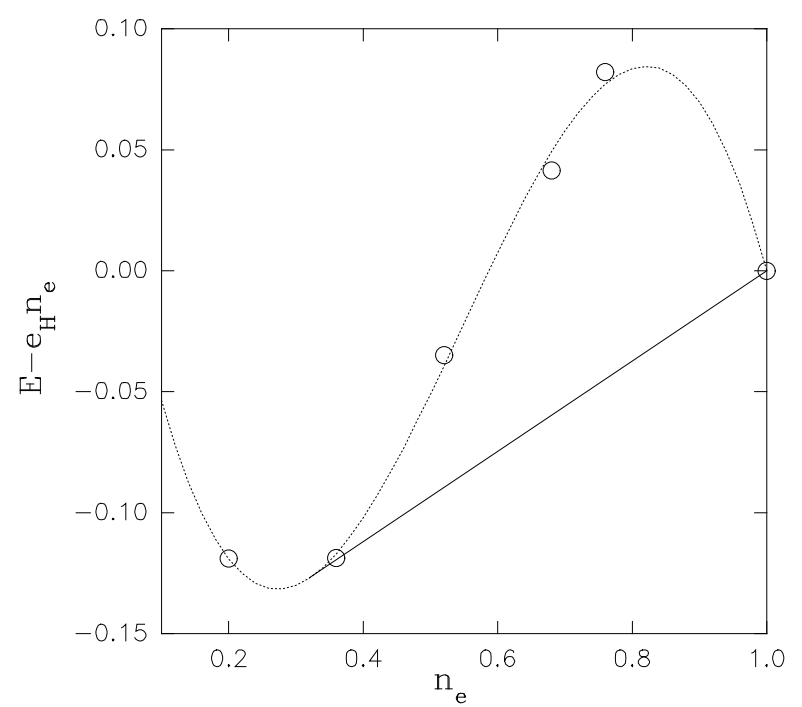

FIG. 7. The ground state energies of different electron densities in 50 site lattice for $J / t=2.5$. The energies are subtracted by the perfect PS energy $e_{H} n_{e}$. The straight line shows the ground state energy of the PS state by Maxwell construction. The onset of PS state is at $n_{e}=0.320$.

Fig.7 shows the energy-density curve of $J / t=2.5$ in 50 sites. The energies are subtracted by $e_{H} n_{e}$, where $e_{H}$ is the Heisenberg energy and $n_{e}$ the electron density. $e_{H} n_{e}$ is energy of the "perfect phase separation state", which separates into a hole-free Heisenberg antiferromagnet and an electron-free vacuum phases. This is the ground state of the infinite $J / t$ limit if the quantum fluctuation near the boundary of the two regions is not taken into account. Thus it is also a good reference state for large $J / t$ cases.

Fig. 7 shows the PS phase boundary determined by Maxwell construction. It is clear that the curve become convex for the larger densities and the phase separates into a hole-free Heisenberg antiferromagnet and a region with density $n_{e}=0.320$. The energy of the PS state is the linear combination of the two phases, which is represented by the solid line. The results are consistent with Hellberg et al.23, whose critical $n_{e}=0.296$.

For smaller $J / t$, it is more difficult to define the tangent line to find the critical $n_{e}$ as we did in Fig.7. The $J / t=0.6$ data are shown in Fig.8(a). It can be seen that the energy-density curve is almost a straight line for this coupling strength. The curve is fitted by a linear function, which is shown by the dotted line in Fig.8(a). And the deviations of the data points from the line are shown in Fig.8(b). We see that the deviations are of the order of magnitude of the statistical error bars. Since the subtraction of a linear function won't change the convexity 

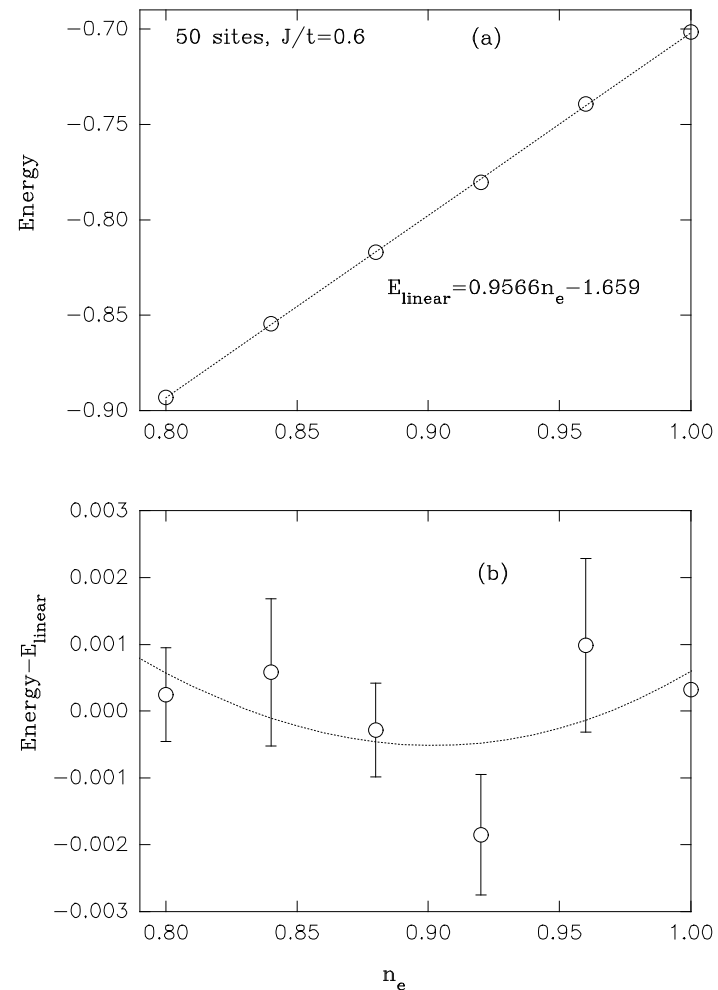

FIG. 8. (a) Energy-density curve for $J / t=0.6$ in 50 sites, it can be fitted very well by a straight line. (b) Deviation of the energy from the line in (a), the values are of the same magnitude of the error bars.

or concavity of the curve, the Maxwell construction can be applied on the curve in Fig.8(b) as we did in Fig.7. A polynomial is used to fit the $E-E_{\text {linear }}$ points. The curve is always concave and there is no PS at this $\mathrm{J} / \mathrm{t}$. An interesting point is that one can always find another fitting function gives PS because the $E-E_{\text {linear }}$ value of $n_{e}=0.96$ is larger than those of $n_{e}=0.92$ and $n_{e}=1$. Since the $E-E_{\text {linear }}$ values are all so small, the fitting is somewhat arbitrary within the error bars. And the energy-density curve is almost linear. It is very likely that $J / t=0.6$ is just the PS phase boundary of vanishing hole density for 50 sites.

Another possibility is the formation of stripe state suggested by S. R. White et al. 42 . For the stripe state, the holes adjust themselves to form stripes to minimize the total energy and does not have the problem of the surface energy as in the PS state. The adjustment makes the energy-density curves linear. But since we use the uniform trial wave functions and periodic boundary condition here, it is difficult to get signals of the stripes state. This will be our future work.

From the two previous examples, we see that it is difficult to read out the slope variation from the energydensity plots to do the Maxwell construction, especially for small $J / t$ values. For such $J / t$, the possible critical $n_{e}$ of PS (if there is) is close to 1 . In this regime, the energy-density curve is very close to a straight line and the ground state energies are very difficult to calculate (or estimate) accurately. It will be quite unreliable to estimate the critical $n_{e}$ and to judge whether there is PS or not by extrapolation and curve fitting. Therefore we will follow Emery et al. 1 by examining a more well-defined value $e(x)$, the energy per hole for hole density $x$.

The energy of the PS state can be separated into two parts:

$$
E \times N_{s}=\left(N_{s}-N\right) e_{H}+N e_{h}
$$

where $N_{s}$ is the total number of sites and $\mathrm{N}$ is the number of sites in the hole-rich phase. The first term of the righthand side of Eq.(37) is the energy of the hole-free Heisenberg region and the second term is that of the hole-rich part $e_{H}=-1.169 \mathrm{~J}$ denotes the Heisenberg energy per site 43 . And $e_{h}$ is energy per site in the uniform hole-rich phase, which is a function of the hole density $x=N_{h} / N$. $N_{h}$ is the number of holes. $E$ can be rearranged into the form:

$$
E \times N_{s}=N_{s} e_{H}+N_{h} e(x)
$$

where

$$
e(x) \equiv\left[-e_{H}+e_{h}(x)\right] / x
$$

$e(x)$ can be interpreted as the energy per hole relative to the half-filled Heisenberg state. If $\mathrm{e}(\mathrm{x})$ of a particular $J / t$ has a minimum at $x=x_{m}$ and the hole density of the total system is smaller than $x_{m}$, the system will adjust the size of the hole-rich phase $\mathrm{N}$ such that $N_{h} / N$ is equal to $x_{m}$ and it minimizes the total energy in Eq.(38). Since $N_{s}, e_{H}$, and $N_{h}$ are all constants, the total energy is minimized as $e(x)$ is minimized. Thus $x_{m}$ is the critical density for phase separation at this $J / t$.

We calculated $e(x)$ from the energy of the uniform states $e_{h}(x)$ by the PL method and found the minimum of $e(x)$ on several sizes of lattices for several densities and $J / t$. The largest one is the $\sqrt{122} \times \sqrt{122}$ cluster. It is very difficult to get the converged ground state energy in the physical regime due to the sign problem. But from the systematic PL procedure, we can see the trend of the change of $e(x)$ and estimate the boundary of the PS state. The PL- 1 power $=4$ (for 82 and 122 sites) or PL-1 power $=6$ (for 50 and 36 sites) energy is used here as the $e_{h}(x)$. It is about $2 \sim 4$ percent lower than the variational energy. We estimate the difference between the best PL energy is within one or two percentage of the true ground state energy.

In Fig. 9 we show the $e(x)$ of $J / t=2.5$ in 50 sites corresponding to Fig.7. The data are fitted by a polynomial and the minimum of $e(x)$ is at $x=0.68$, which is exactly agree with the density $n_{e}=0.32$ determined from Fig.7. Thus the two versions of Maxwell construction are consistent. The advantage of the latter one is that the critical density for PS state will be determined much more accurately if the energy-density curve is almost linear. This is the case for the physical interested regime. So we will use the $e(x)$ to determine the PS boundary in this report. 


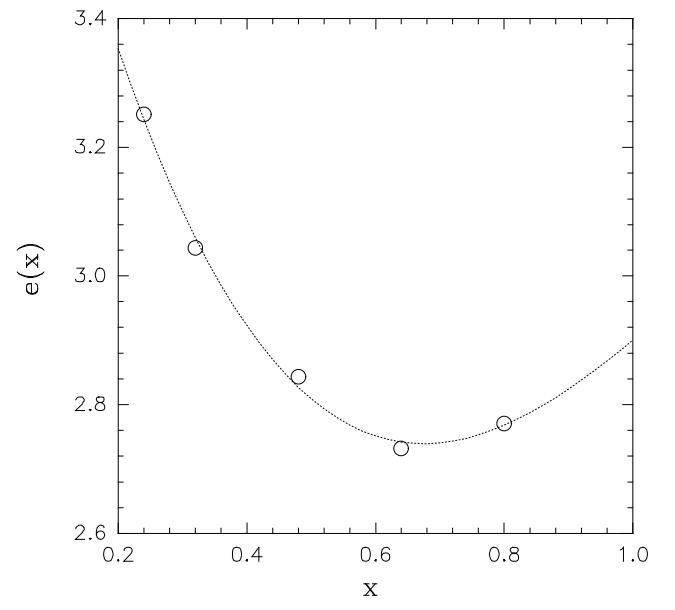

FIG. 9. $e(x)$ vs hole density $x$ of $J / t=2.5$ in 50 sites. The minimum of the fitted curve is at $x=0.68$.

\section{RESULTS AND DISCUSSION}

Using the Maxwell construction we determined the phase boundary of PS state for several $J / t$. The results are shown in Fig.10. The results evaluated by using Green function Monte Carlo with stochastic reconfiguration (GFMCSR) by M. Calandra et al 44 and freen function Monte Carlo with extrapolation by $\mathrm{HM}^{23}$ are also shown in the figure.

From Fig.10 we see that in the physical regime, our data are consistent with the GFMCSR results that there is a lower bound of the $J / t$ value for PS state. This is also the case in our previous study 45 . In contrast, the extrapolated GFMC results show that PS state occurs at all values of $J / t$. In the large $J / t$ and low electron density region, all the results are qualitatively consistent.

There are several possible reasons for the inconsistence in the physical region. First, the energies calculated are not accurate enough. The energy-density curve is very close to a straight line in this region, so a small error in energy will cause a completely different result of Maxwell construction because the method is related to the second derivative of the curve. Fig.11 shows the PS phase boundaries determined by energies evaluated by different projection levels. It is clear that as the powers increase (that is, the energies closer to the ground state energies), the PS phase boundary will be pushed to larger $J / t$ value and electron densities. This feature appears in all of our calculation on different lattices. It is strongly suggestive that the PS phase boundary determined from our variational calculation is a lower bound of $\mathrm{J} / \mathrm{t}$ and electron densities of the real boundary.

In TABLE II we show that our PL energies are lower than the extrapolated GFMC values. And since PL method is a variational one, the true ground state energies will be even lower. We use $J / t=0.3$ in 122 sites as an example to demonstrate the effect of the non-converged energies in more detail. Again the energy-density plot

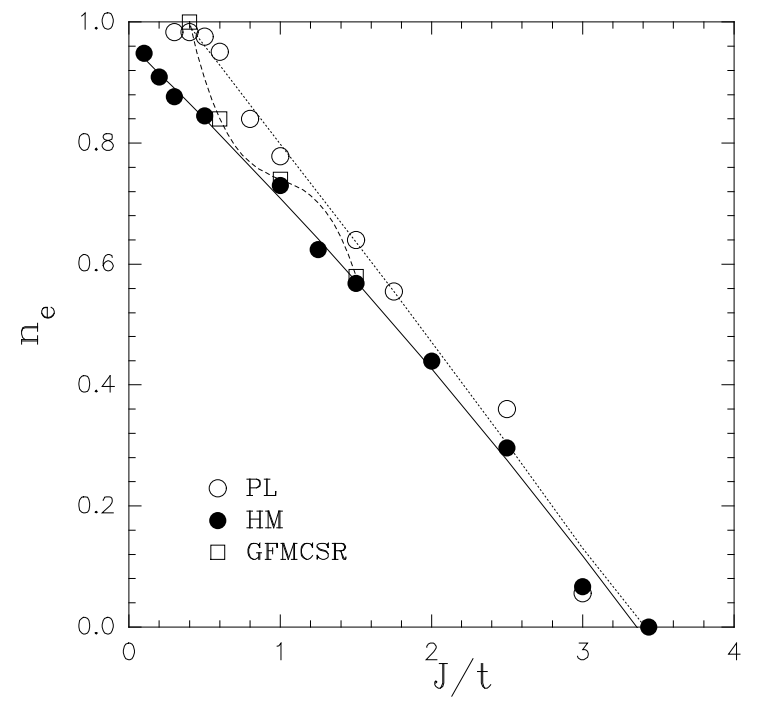

FIG. 10. Phase separation boundary on the phase diagram of the two-dimensional $t-J$ model evaluated by the power-Lanczos method (open circles), GFMCSR method by M. Calandra et al. (open squares), and GFMC with extrapolation by C. S. Hellberg et al. (full circles).

in Fig.12(a) is very close to a straight line and it will be dangerous to fit the curve and draw the tangent line from the half-filled point.

In Fig.12(b) we show the $e(x)$ vs. hole density $x$ curves for different level of projection. As the power increases, the energy decreases and approaches to the ground state energy, and $e(x)$ also decreases. It is interesting that $e(x)$ of smaller $x$ drops much faster than those of large $x$. In this case, at the $\mathrm{PL}_{\text {power }}=0$ level, the minimal $e(x)$ will occur at $x$ larger than 0.0656 ( 8 holes in 122 sites). While at the PL1 $1_{\text {power }=0}$ level, the curve becomes almost flat. For PL $1_{\text {power }=4}$, the smallest density $x=0.0164$ ( 2 holes) becomes the lowest one. Note that the energies have not yet converged. From the trend of $e(x)$ with increasing power, we expect the minimum of $e(x)$ at $x=0.0164$ will be even deeper. That is, the PS state occurs at $x \leq 0.0164$, while a much larger value $x_{P S}=0.123$ is reported by HM.

The changes of energies and $e(x)$ with respect to powers are shown in Fig.13. It is clear that $e(x)$ drops faster for at lower hole densities with increasing powers. The reason is that the hole density is at the denominator of the definition of $e(x)$. From the curves in Fig.13, it is clear that the energies have not converged yet. And the trend of the curves strongly suggests that the $e(x)$ of the smaller $x$ cases will be much more reduced than those of the larger ones. That is, if the ground state energies are calculated more accurately, the PS boundary will be pushed toward the higher density or larger $J / t$. Thus the true phase boundary will be at the upper right side of our variational result in Fig.10.

Fig.14 shows the similar plots for $J / t=0.4$. The behavior is a little subtle in this value of $J / t$. The $e(x)$ is 


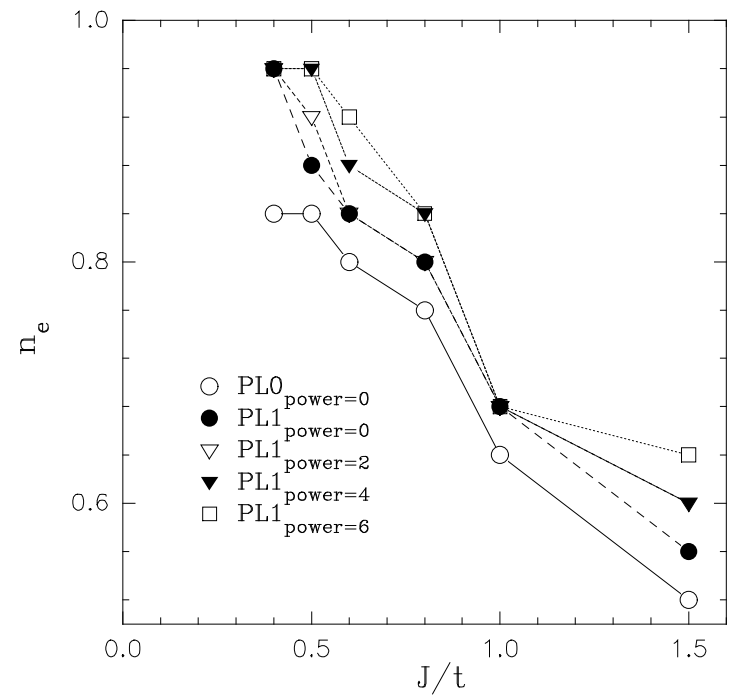

FIG. 11. PS phase boundaries determined by different PL levels. It can be seen that for larger powers, the boundary is pushed to larger $J / t$ and electron density $n_{e}$.

flatter than the $J / t=0.3$ case. The minimal value is also at $x=0.0164$ point, but within error bar with the $x=0.0328$ ( 4 holes) point. Again the energies are still well above the ground state values, we expect the $e(x)$ for $x=0.0164$ will be even lower.

Note that in Fig.12(b) and Fir 14.(b) the Heisenberg energy used is the thermodynamic limit $e_{H}=-1.169 \mathrm{~J}$. And $e_{H}=-1.1713 J$ for finite system with 122 sites are used for Fig.12(c) and Fig 14.(c). The later case will make the $e(x)$ values larger. And in the $J / t=0.4$ case, the PL1 $1_{\text {power }=4}$ curve becomes almost flat. This finite-size effect will shift the phase boundary a little but the main results will be unchanged.

Another way to understand the finite-size effect is to use finite-size scaling. For example, in the case of $J / t=0.4$ in 98 sites shown in Fig.20 in 23 (calculated by M. Calandra et al.), the minimum of $e(x)$ is at the $x_{m}=0.041$ ( 4 holes) point. It is possible that PS occurs at this hole density. But together with the 50 site data in the same figure, the $x_{m}=0.08$, also for 4 holes. The $x_{m}$ becomes a half as the size of lattice is doubled. This scaling behavior is consistent with that reported in our previous paper 4 . And the scaling behavior to even larger lattices is also shown in Fig.3 of Ref 44 . Note that all the energies used in these references are upper bounds of the ground state energies. We expect that $e(x)$ for smaller hole density $x$ will become even lower, and the phase boundary of PS state will be pushed to larger electron density and larger $J / t$. Thus in contrast with the conclusion by in Ref23, we conclude that PS does not occur at $J / t=0.4$ for any density.

Another finite size effect comes from the different shell for different electron density. The effect makes the energy-density curves jagged and difficult to analyze. HM calculated the energies for the same number

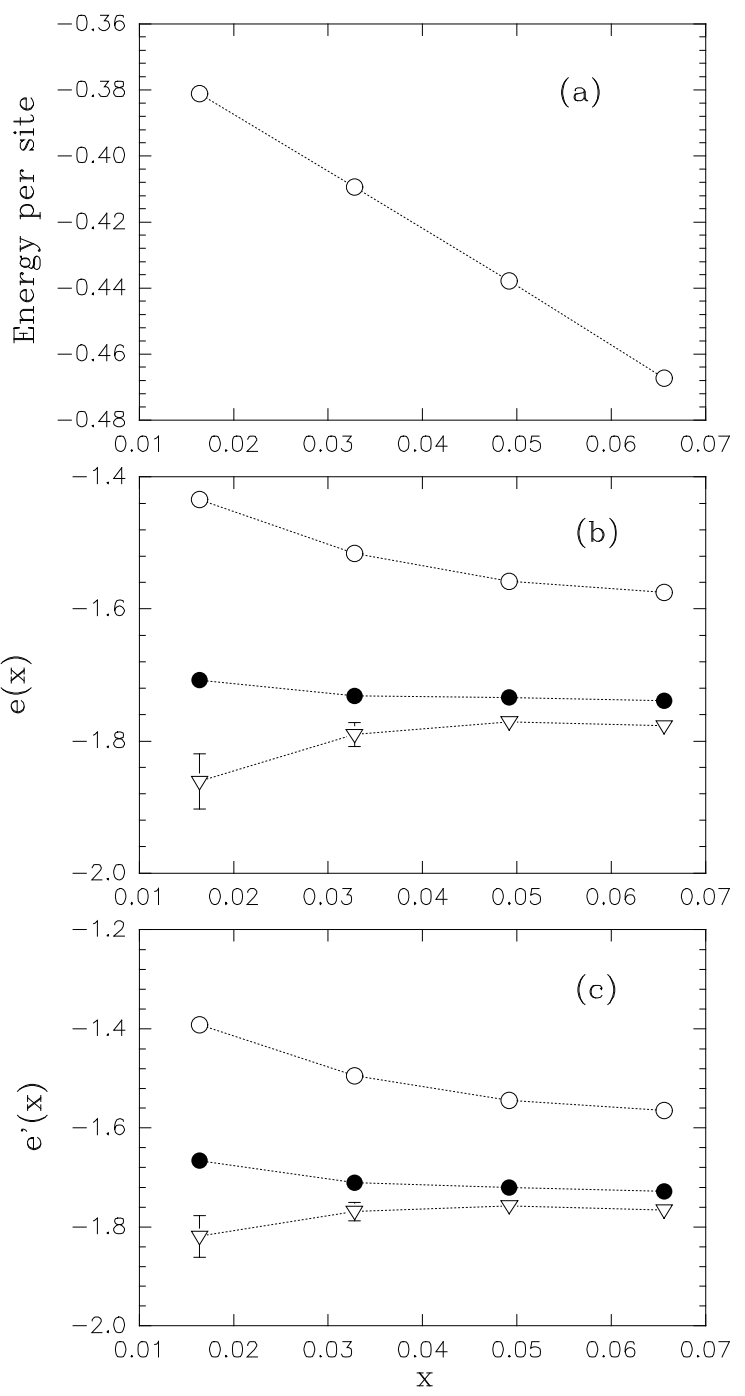

FIG. 12. (a)Energy vs. hole density $x$ for $J / t=0.3$ in 122 sites. (b) $\mathrm{PL} 0_{\text {power }=0}$ (open circle), $\mathrm{PL} 1_{\text {power }=0}$ (full circle), and $\mathrm{PL} 1_{\text {power }=4}$ (open triangle) $e(x)$ calculated by using $e_{H}=-1.169 J$ and $(\mathrm{c}) e_{H}=-1.1713 J$.

of electrons (with the same filled shell) in different sizes of lattices and different boundary conditions to avoid this effect. But there are still other finite size effects in this way due to the different sizes and boundary conditions. The only way to eliminate the finite size effects is to study this problem in different, and large enough sizes of lattices.

For $J / t \leq 0.4$, the minimal $e(x)$ is always at two holes for lattices of different sizes (up to 122) This may be resulted from the two-hole bound state 27 but not PS at $x_{m}=2 / N_{s}$. If there were PS, $x_{m}$ would be at the same (or nearby) density rather than the same number of holes.

In conclusion, from the analysis of the energies evaluated from the PL method, we conclude that $J / t=0.4$ is a lower bound for PS. The phases will not separate for $J / t$ smaller than this value. From the trend of the 


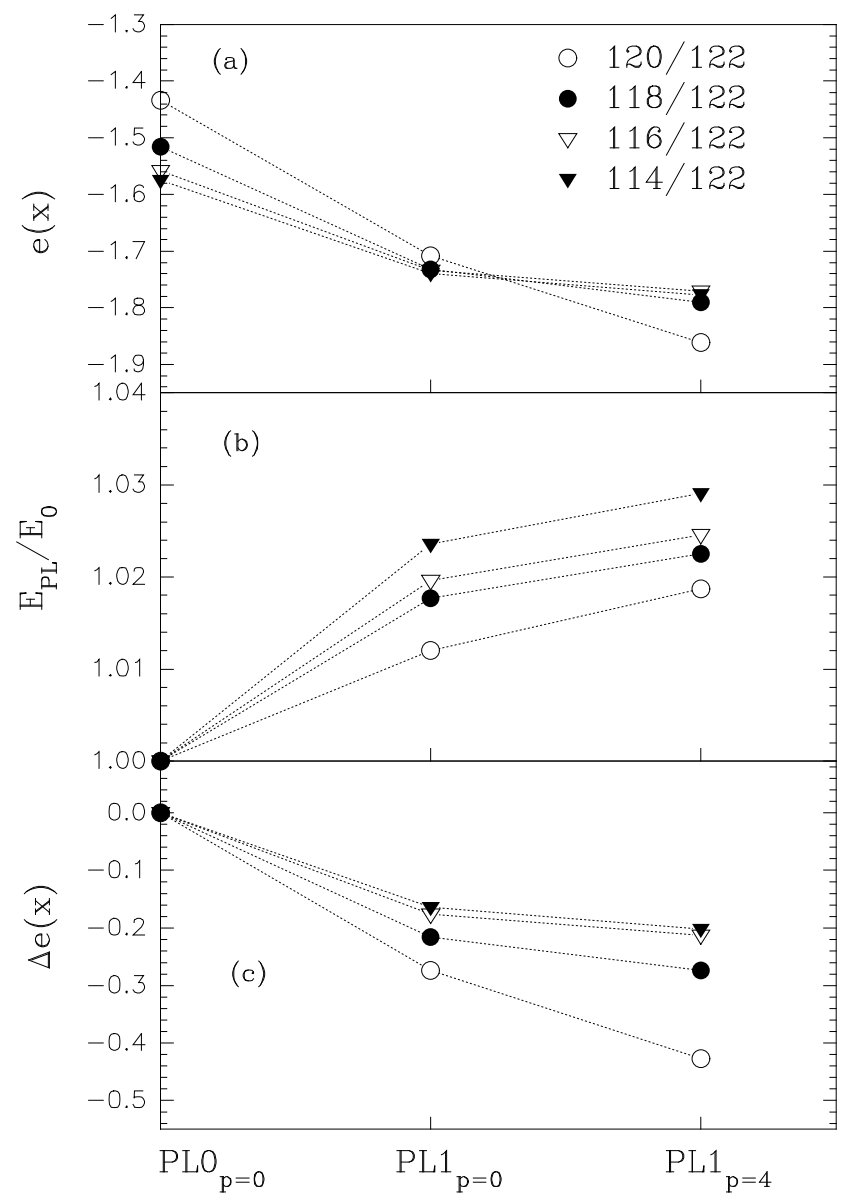

FIG. 13. (a) e(x) of $J / t=0.3$ for different powers in 122 sites. The electron numbers are 120 (open circles), 118 (full circles), 116 (open triangles), and 114 (full triangles). (b)The ratios of power energies $E_{P L}$ and variational $\left(P L 0_{\text {power }=0}\right)$ energy $E_{0}$. (c)The differences between $e(x)$ of different powers and the variational value.

energies and $e(x)$ functions, this lower bound may be pushed to even larger $J / t$. The extrapolation of ground state energies and energy-density curves may be unreliable and misleading in the physical interesting regime. F. Becca et al. give upper and lower bounds for the ground state energy of the infinite- $U$ 2D Hubbard model, which is equivalent to the $J / t=0$ case for the $t-J$ model. Their analysis on this case ruled out the possibility of PS for the electron density less than half-filled 1 . By the way, from the finite size analysis of the exact diagonalization data for one and two holes in smaller clusters (up to 32 sites) gives the results that there is no two-hole bound state for $J / t \leq 0.640 .42 .8$. Thus it seems the attractive interaction is not strong enough to be the mechanism to cause the PS state for small $J / t$ values.

We thank M. Calandra, S. Sorella, and P. W. Leung for their kindness to give us their data and very useful discussions. This work is supported by the National Science Council of Republic of China, Grants No. NSC89-2112M-001-103, NSC89-2112-M-029-004, and NSC89-2112-
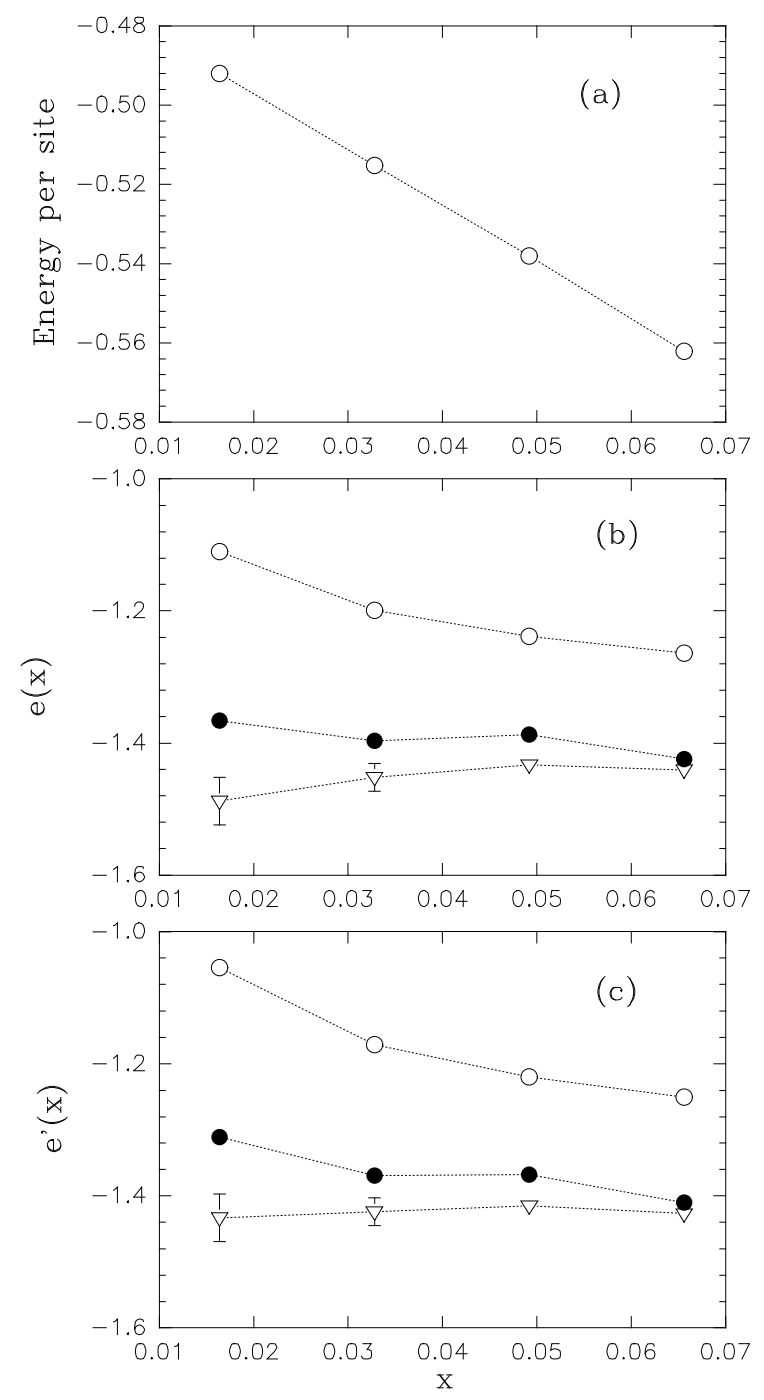

FIG. 14. (a)Energy vs. hole density $x$ for $J / t=0.4$ in 122 sites. (b) $\mathrm{PL} 0_{\text {power }=0}$ (open circle), $\mathrm{PL} 1_{\text {power }=0}$ (full circle), and $\mathrm{PL} 1_{\text {power }=4}$ (open triangle) $e(x)$ calculated by using $e_{H}=-1.169 J$ and $(\mathrm{c}) e_{H}=-1.1713 J$.

M-321-001. Part of the computations were performed on the IBM SP2 Power3 and PC Linux cluster of the National Center for High-Performance Computing in Taiwan. We are grateful for their support.

${ }^{1}$ V. J. Emery, S. A. Kivelson, and H. Q. Lin, Phys. Rev. Lett. 64, 475 (1990).

2 J. M. Tranquada, B. J. Sternlieb, J. D. Axe, Y. Nakamura, S. Uchida, Nature 375, 561 (1995).

${ }^{3}$ P. Prelovšek, and X. Zotos, Phys. Rev. B 47, 5984 (1993).

${ }^{4}$ S. R. White and D. J. Scalapino, Phys. Rev. Lett. 80, 1272 (1998). 
${ }^{5}$ S. R. White and D. J. Scalapino, Phys. Rev. Lett. 81, 3227 (1998).

${ }^{6}$ E. Dagotto et al., Phys. Rev. B 49, 3548 (1994).

${ }^{7}$ V. J. Emery, and S. A. Kivelson, Physica C 209, 597 (1993).

${ }^{8}$ J. D. Jorgensen et al, Phys. Rev. B 38, 11337 (1988).

${ }^{9}$ P. C. Hammel et al., Phys. Rev. B 42, 6781 (1990)

${ }^{10}$ P. C. Hammel et al., Physica C 185-189, 1095 (1991).

${ }^{11}$ F. C. Chou et al., Phys. Rev. B 54, 572 (1996).

12 X. L. Dong et al., Phys. Rev. Lett. 80, 2701 (1998).

13 J. H. Cho et al., Phys. Rev. Lett. 70, 222 (1993).

14 A. Weidinger, Ch. Niedermayer, A. Golnik, R. Simon, and E. Recknagel, Phys. Rev. Lett. 62, 102 (1989).

${ }^{15}$ D. R. Harshman, G. Aeppli, B. Batlogg, G. P. Espinosa, R. J. Cava, A. S. Copper, L. W. Rupp, E. J. Ansaldo, and D. Ll. Williams, Phys. Rev. Lett. 63, 1187 (1989).

${ }^{16}$ J. H. Cho et al., Phys. Rev. B 46, 3179 (1992).

${ }^{17}$ F. Borsa et al., Physica C 235-240, 1713 (1994).

${ }^{18}$ F. Borsa et al., Phys. Rev. B 52, 7334 (1995).

${ }^{19}$ E. G. Nikolaev, H. B. Brom, and A. A. Zakharov, Phys. Rev. B 62, 3050 (2000).

${ }^{20}$ H. H. Wen, X. H. Chen, W. L. Yang, and Z. X. Zhao, Phys. Rev. Lett. 85, 2805 (2000).

${ }^{21}$ C. S. Hellberg, and E. Manousakis, Phys. Rev. B 52, 4639 (1995).

22 C. S. Hellberg, and E. Manousakis, Phys. Rev. Lett. 78, 4609 (1997).

${ }^{23}$ C. S. Hellberg, and E. Manousakis, Phys. Rev. B 61, 11787 (2000).

24 Tae-Hyoung Gimm, and Sung-Ho Suck Salk, Phys. Rev. B62, 13930 (2000).

${ }^{25}$ A. Moreo et al., Phys. Rev. B 43, 11442 (1991).

${ }^{26}$ F. Becca, M. Capone, and S. Sorella, Phys. Rev. B 62, 12700 (2000).

${ }^{27}$ E. Dagotto et al., Phys. Rev. B 45, 10741 (1992).

${ }^{28}$ W. O. Putikka, M. U. Luchini, and T. M. Rice, Phys. Rev. Lett. 68, 538 (1992).

${ }^{29}$ W. O. Putikka, and M. U. Luchini, Phys. Rev. B 62, 1684 (2000).

${ }^{30}$ D. Poilblanc, Phys. Rev. B 52, 9201 (1995).

${ }^{31}$ H. Yokoyama and M. Ogata, J. Phys. Soc. Jpn 65, 3615 (1996).

${ }^{32}$ Y. C. Chen, and T. K. Lee, Phys. Rev. B51, 6723 (1995).

${ }^{33}$ E. S. Heeb, and T. M. Rice, Z. Phys. B 90, 73 (1993).

${ }^{34}$ M. Khono, Phys. Rev. B 55, 1435 (1997).

${ }^{35}$ W. von der Linden, Phys. Rep. 220, 53 (1992).

36 T. K. Lee, and Shiping Feng, Phys. Rev. B38, 11809 (1988).

${ }^{37}$ D. Ceperley, G. V. Chester, and M. H. Kalos, Phys. Rev. B16, 3081 (1977).

${ }^{38}$ E. S. Heeb, and T. M. Rice, Europhys. Lett. 27, 673 (1994).

${ }^{39}$ P. W. Leung, and R. J. Gooding, Phys. Rev. B52, 15771 (1995).

${ }^{40}$ C. T. Shih, Y. C. Chen, H. Q. Lin, and T. K. Lee, Phys. Rev. Lett. 81, 1294 (1998).

${ }^{41}$ C. S. Hellberg, and E. J. Mele, Phys. Rev. B 48, 646 (1993).

${ }^{42}$ S. R. White, and D. J. Scalapino, Phys. Rev. B61, 6320 (2000).

43 A. Sandvik, Phys. Rev. B 56, 11678 (1997).

${ }^{44}$ M. Calandra, F. Becca, and S. Sorella, Phys. Rev. Lett. 81, 5185 (1998).
${ }^{45}$ C.T. Shih, Y.C. Chen, and T.K. Lee, Phys. Rev. B57, 627 (1998).

${ }^{46}$ F. Becca, L. Capriotti, and S. Sorella, cond-mat/0009359 (2000).

47 D. Poilblanc, Phys. Rev. B48, 3368 (1993).

48 A. L. Chernyshev, P. W. Leung, and R. J. Gooding, Phys. Rev. B58, 13594 (1998). 